\title{
Influence of Some Constituents of Serum on Gas Metabolism of Tissue in vitro.
}

\author{
III Report.
}

Influence of Sodium Lactate upon Tissue Respiration in vitro.

By

Hitosi Yamamoto.

(山本均)

(From the Institute ofPhysiology, Director: Prof. S. Kodama, Kumamoto Medical College.)

Relation between the respiration and the formation or breakdown of lactic acid in animal bodies has been extensively studied in muscle tissue. But the experiment was formerly made on whole animal or organs.

Warburg's ${ }^{122) 3}$ manometric method for surviving tissue slices has given us a more precise means for studying metabolic processes in tissues.

The method was first applied by Meyerhof ${ }^{455}$ and his collaborators for investigating influence of lactic acid on tissue respiration. They found that addition of lactate to Ringe r's solution caused increase of the respiration of various tissues such as muscle, liver, kidney and others. They considered further that the action of serum in increasing the tissue respiration was due to presence of lactate in the serum. L o e b e $\mathrm{l}^{\text {b }}$ also confirmed the results of the above investigators in his experiment on brain tissue.

We confirmed in our first report ${ }^{7}$ that the active factors which effected an increase of tissue respiration in serum had to be diffusible matters and then found glucose as one of the diffusible effective substances.

1) Warburg, O.; Biochem. Z., 1923, 142, 317.

2) Id.; ibid., 1924, 152, 51.

3) Id.; ibid., 1925, 164, 481 .

4) Meyerh of, O., K. Lohmann and R. Meier; Biochem. Z., 1925, 157, 459.

5) Me yerh of, O. and K. Lohm a n n; Biochem. Z., 1926, 171, 381.

6) Is o bel, R. Q. ; Biochem. Z., 1925, 161, 219.

7) Y a ma mot o, H. ; Tohoku J. Exp. Med., 1938, 33, 454. 
As the second effective substance lactate was taken into consideration and was experimented on in reference to its influence on tissue respiration of kidney cortex.

\section{Method.}

The method for measurement of oxygen consumption and carbon dioxide output of the tissues was Warburg's second one.2) Tissue used was cortical one of rabbit kidney. Rabbit was starved 24 hours before the experiment. The tissue was perfused with the normal Ringer's solution') (containing $0.2 \%$ glucose) before removal of the kidneys. The kidney was kept in the same Ring er's solution before and after having been dissected into thin slices. Gas for the experiments was 3.5 per cent carbon dioxide in oxygen. The $\mathrm{pH}$ of the solution suspending tissue slices in experimental vessels was 7.32 when saturated with this gas. Manometric readings were taken every 20 minutes during two hours and $\mathrm{Q}$ o and $\mathrm{Qc}$ were calculated for each 20 minutes.

Ringer's solutions containing 10,20 and $60 \mathrm{mgm}$ per cent sodium lactate (Katayama, chemical pure.) were prepared, and tissue respiration in these solutions were compared with that in the normal Ringer's solution.

The series of experiments were done simultaneously with the tissue slices from the same kidney. Ringer's solutions suspending tissue slices contained in one series the above cited concentrations of lactate and in the other series no lactate. The latter series served as a contról experiment for the former.

\section{Experiment.}

1. Effect of $10 \mathrm{mgm}$ per cent sodium lactate in Ringer's solution upon tissue respiration of kidney cortex in vitro.

Table 1 (Fig. 1) is the protocol of this experiment.

The means of the oxygen consumption Qo and carbon dioxide output $\mathrm{Qc}$ of Table $1 \mathrm{a}$ for two hours were 18.3 and $15.9 \mathrm{cmm}$ respectively, of which the means for the first hour were 20.3 and $17.6 \mathrm{cmm}$ and those for the second 16.5 and $14.1 \mathrm{cmm}$.

The means of Qo and Qc of Table $1 \mathrm{~b}$ (control experiment for $1 \mathrm{a}$ ) for two hours were 14.9 and $12.6 \mathrm{cmm}$ respectively, of which the means for the first hour were 16.0 and $13.6 \mathrm{cmm}$ and those for the second 13.7 and $11.5 \mathrm{cmm}$. 
The increase of Qo by addition of $10 \mathrm{mgm}$ per cent sodium lactate to Ringer's solution was $23 \%$ for two hours, that for the first and second hour was $27 \%$ and $20 \%$ respectively. The increase of $\mathrm{Qc}$ by addition of lactate was $26 \%$ for two hours, that for the first and second hour was $29 \%$ and $23 \%$ respectively.

There was no appreciable difference between the R.Q. by addition of lactate.

\section{Effect of 20 mgm per cent sodium lactate in Ringer's solution} upon tissue respiration of kidney cortex in vitro.

Influence of sodium lactate of $20 \mathrm{mgm}$ per cent was investigated.

The means of the oxygen consumption Qo and carbon dioxide output Qc of Table 2 a were 18.9 and $15.7 \mathrm{cmm}$ respectively for two hours, of which the means for the first hour were 19.9 and $16.9 \mathrm{cmm}$ and those for the second 17.9 and $14.8 \mathrm{cmm}$.

The means of Qo and Qc of Table $2 \mathrm{~b}$ (control experiment for Table $2 \mathrm{a})$ were 17.6 and $15.5 \mathrm{cmm}$ respectively for two hours, of which the means for the first hour were 20.3 and $18.3 \mathrm{cmm}$ and those for the second 15.0 and $12.6 \mathrm{cmm}$.

The increase of Qo in the presence of $20 \mathrm{mgm}$ per cent sodium lactate in Ring er's solution was $7 \%$ for two hours; for the first hour a slight decrease was appreciable, however, and for the second hour the increase was $19 \%$.

The increase of $\mathrm{Qc}$ by addition of $20 \mathrm{mgm}$ per cent lactate for two hours was $3 \%$; for the first hour the decrease was appreciable (8\%), but for the second hour the increase was found to be $17 \%$.

In this case the effect of sodium lactate became with the lapse of time evident.

R. Q. were rather higher in the normal Ringer's solution for the first hour.

3. Effect of $60 \mathrm{mgm}$ per cent sodium lactate in Ringer's solution upon tissue respiration of kidney cortex in vitro.

Sodium lactate was added in concentration of $60 \mathrm{mgm}$ per cent to Ring er's solution and the tissue respiration in this solution was compared with that in the normal Ringer's solution.

Results were shown in Table 3 (Fig. 3). 
TABLE

Effect of 10 mgm per cent sodium lactate in Ringer's

(a) Tissue respiration of rabbit kidney cortex in Ringer's

\begin{tabular}{|c|c|c|c|c|c|c|c|c|c|c|}
\hline \multirow{2}{*}{ No. } & \multirow{2}{*}{ Date } & \multicolumn{6}{|c|}{ Qo } & \multicolumn{3}{|c|}{$Q$} \\
\hline & & $\underset{20}{I}$ & $\begin{array}{l}\text { II } \\
20\end{array}$ & $\begin{array}{c}\text { III } \\
20\end{array}$ & $\begin{array}{l}\text { IV } \\
20\end{array}$ & $\begin{array}{c}V \\
20\end{array}$ & $\begin{array}{l}\text { VI } \\
\mathbf{2 0}\end{array}$ & $\underset{20}{I}$ & $\begin{array}{l}\text { II } \\
20\end{array}$ & $\underset{20}{\text { III }}$ \\
\hline 1 & $23 / 6$ & 38.4 & 31.8 & 30.9 & 27.0 & 23.3 & 21.1 & 38.7 & 32.3 & 30.8 \\
\hline 2 & $24 / 6$ & 22.0 & 17.1 & 19.0 & 16.3 & 10.9 & 17.8 & 19.6 & 14.1 & 16.2 \\
\hline 3 & $25 / 6$ & 20.2 & 17.3 & 16.3 & 15.1 & 15.2 & 15.7 & 16.2 & 13.6 & 13.0 \\
\hline 4 & $26 / 6$ & 20.6 & 13.1 & 11.6 & 24.5 & 17.2 & 12.3 & 20.1 & 9.4 & 8.6 \\
\hline 5 & $27 / 6$ & 29.9 & 18.2 & 15.5 & 16.5 & 15.4 & 13.5 & 28.6 & 15.0 & 13.1 \\
\hline 6 & $30 / 6$ & 18.9 & 19.9 & 20.8 & 17.3 & 15.3 & 16.9 & 14.0 & 16.1 & 17.3 \\
\hline 7 & $1 / 7$ & 21.4 & 17.4 & 15.6 & 14.1 & 15.6 & 7.8 & 19.6 & 14.4 & 12.6 \\
\hline 8 & $1 / 7$ & 14.5 & 22.8 & 15.4 & 16.0 & 13.4 & 16.6 & 10.0 & 17.9 & 11.9 \\
\hline & Mean & 23.2 & 19.7 & 18.1 & 18.4 & 15.8 & 15.2 & \begin{tabular}{|l|}
20.9 \\
\end{tabular} & 16.6 & 15.4 \\
\hline & & \pm 1.74 & \pm 1.23 & \pm 1.30 & \pm 1.19 & \pm 0.71 & \pm 0.96 & \pm 2.05 & $\pm 1.36^{2}$ & \pm 1.43 \\
\hline
\end{tabular}

(b) Tissue respiration of rabbit kidney cortex in

\begin{tabular}{|c|c|c|c|c|c|c|c|c|c|c|}
\hline \multirow{2}{*}{ No. } & \multirow{2}{*}{ Date } & \multicolumn{6}{|c|}{ Qo } & \multicolumn{3}{|c|}{$Q$} \\
\hline & & $\begin{array}{c}\text { I } \\
20\end{array}$ & $\begin{array}{l}\text { II } \\
\mathbf{2 0}\end{array}$ & $\begin{array}{c}\text { III } \\
20\end{array}$ & $\begin{array}{l}\text { IV } \\
20\end{array}$ & $\begin{array}{c}V \\
20\end{array}$ & $\begin{array}{l}\text { VI } \\
20\end{array}$ & $\underset{20}{I}$ & $\begin{array}{l}\text { II } \\
20\end{array}$ & $\begin{array}{c}\text { III } \\
20\end{array}$ \\
\hline 1 & $23 / 6$ & 9.0 & 10.5 & 9.8 & 11.6 & 12.1 & 12.7 & 6.8 & 8.9 & 7.4 \\
\hline 2 & $24 / 6$ & 19.6 & 16.1 & 13.7 & 16.3 & 11.4 & 16.3 & 18.1 & $\begin{array}{r}0.6 \\
13.6\end{array}$ & 11.0 \\
\hline 3 & $25 / 6$ & 24.1 & 19.1 & 17.3 & 14.3 & 15.5 & 15.6 & 22.9 & 17.1 & 15.0 \\
\hline 4 & $26 / 6$ & 15.0 & 10.1 & 16.5 & 15.9 & 15.4 & 13.0 & 12.6 & 6.5 & 13.3 \\
\hline 5 & $27 / 6$ & 21.4 & 17.2 & 19.7 & 17.9 & 16.8 & 14.7 & 18.7 & 15.2 & 18.2 \\
\hline 6 & $30 / 6$ & 13.8 & 17.0 & 14.6 & 13.1 & 10.0 & 14.2 & 11.2 & 14.0 & 12.0 \\
\hline 7 & $1 / 7$ & 18.1 & 17.1 & 11.5 & 10.0 & 12.6 & 9.1 & 17.8 & 15.3 & 8.7 \\
\hline 8 & $1 / 7$ & 22.3 & 19.1 & 11.6 & 13.9 & 12.0 & 15.2 & 19.3 & 15.4 & 7.7 \\
\hline & Mean & 17.9 & 15.8 & 14.3 & 14.1 & 13.2 & 13.9 & 15.9 & 13.3 & 11.7 \\
\hline & & \pm 1.27 & \pm 0.87 & \pm 0.86 & \pm 0.63 & \pm 0.64 & \pm 0.54 & \pm 1.37 & \pm 0.88 & \pm 0.95 \\
\hline
\end{tabular}

TABLE

Effect of 20 mgm per cent sodium lactate in Ringer's

(a) Tissue respiration of rabbit kidney cortex in Ringer's

\begin{tabular}{|c|c|c|c|c|c|c|c|c|c|c|}
\hline \multirow{2}{*}{ No. } & \multirow{2}{*}{ Date } & \multicolumn{6}{|c|}{ Qo } & \multicolumn{3}{|c|}{$\mathbf{Q}$} \\
\hline & & $\begin{array}{c}\text { I } \\
20\end{array}$ & $\begin{array}{l}\text { II } \\
20\end{array}$ & $\begin{array}{c}\text { III } \\
20\end{array}$ & $\begin{array}{l}\text { IV } \\
20\end{array}$ & $\begin{array}{c}V \\
20\end{array}$ & $\begin{array}{l}\text { VI } \\
20\end{array}$ & $\begin{array}{c}I \\
20\end{array}$ & $\begin{array}{l}\text { II } \\
20\end{array}$ & $\begin{array}{c}\text { III } \\
20\end{array}$ \\
\hline 1 & $12 / 6$ & 31.3 & 35.4 & 29.1 & 22.2 & 20.9 & 18.9 & 30.2 & 35.6 & 27.1 \\
\hline 2 & $13 / 6$ & 20.9 & 20.2 & 17.4 & 16.1 & 14.7 & 15.4 & 16.7 & 16.0 & 14.0 \\
\hline 3 & $13 / 6$ & 16.3 & 22.1 & 13.4 & 16.5 & 21.0 & 19.1 & 11.9 & 18.1 & 12.2 \\
\hline 4 & $17 / 6$ & 13.1 & 25.3 & 17.1 & 14.1 & 12.4 & 14.3 & 10.6 & 20.1 & 14.1 \\
\hline 5 & $18 / 6$ & 14.1 & 20.3 & 16.0 & 17.1 & 12.7 & 16.8 & 11.6 & 15.7 & 12.4 \\
\hline 6 & $19 / 6$ & 31.6 & 21.2 & 10.0 & 23.8 & 33.0 & 28.5 & 26.4 & 18.9 & 6.4 \\
\hline 7 & $20 / 6$ & 25.2 & 11.4 & 17.7 & 18.0 & 18.8 & 14.3 & 19.0 & 7.4 & 13.0 \\
\hline 8 & $20 / 6$ & 13.9 & 18.0 & 17.4 & 15.1 & 13.5 & 12.8 & 14.0 & 17.6 & 16.2 \\
\hline & Mean & $\begin{array}{r}20.8 \\
\pm 2.06\end{array}$ & $\begin{array}{r}21.7 \\
\pm 1.40\end{array}$ & $\begin{array}{r}17.3 \\
\pm 1.00\end{array}$ & $\begin{array}{r}17.9 \\
\pm 0.83\end{array}$ & $\begin{array}{r}18.4 \\
\pm 1.61\end{array}$ & $\begin{array}{r}17.5 \\
\pm 1.11\end{array}$ & $\begin{array}{r}17.6 \\
\pm 1.84\end{array}$ & $\begin{array}{r}18.7 \\
+1.49\end{array}$ & $\begin{array}{r}14.4 \\
+1.15\end{array}$ \\
\hline
\end{tabular}


1.

solution upon tissue respiration of kidney cortex in vitro. solution with $10 \mathrm{mgm}$ per cent sodium lactate.

\begin{tabular}{|c|c|c|c|c|c|c|c|c|}
\hline & & & \multicolumn{6}{|c|}{ R. Q. } \\
\hline IV & V & VI & I & II & III & IV & V & VI \\
\hline 20 & 20 & 20 & 20 & 20 & 20 & 20 & 20 & 20 \\
\hline 24.7 & 21.2 & 20.0 & 1.01 & 1.02 & 1.00 & 0.92 & 0.91 & 0.95 \\
\hline 13.7 & 8.0 & 15.6 & 0.89 & 0.83 & 0.85 & 0.84 & 0.74 & 0.88 \\
\hline 12.6 & 12.4 & 13.6 & 0.80 & 0.79 & 0.80 & 0.83 & 0.82 & 0.87 \\
\hline 23.2 & 14.9 & 11.0 & 0.98 & 0.72 & 0.74 & 0.95 & 0.87 & 0.90 \\
\hline 14.2 & 13.3 & 11.3 & 0.96 & 0.82 & 0.85 & 0.86 & 0.86 & 0.84 \\
\hline 14.7 & 13.0 & 14.7 & 0.74 & 0.81 & 0.83 & 0.85 & 0.85 & 0.87 \\
\hline 11.4 & 14.9 & 4.2 & 0.92 & 0.83 & 0.81 & 0.81 & 0.96 & 0.54 \\
\hline 13.1 & 9.3 & 13.7 & 0.69 & 0.79 & 0.77 & 0.82 & 0.69 & 0.83 \\
\hline 16.0 & 13.4 & 13.0 & 0.87 & 0.83 & 0,83 & 0.86 & 0.84 & 0.84 \\
\hline \pm 1.29 & \pm 0.87 & \pm 1.00 & +10.03 & \pm 0.02 & \pm 0.02 & \pm 0.01 & \pm 0.02 & \pm 0.02 \\
\hline
\end{tabular}

the normal Ring er's solution.

\begin{tabular}{|c|c|c|c|c|c|c|c|c|}
\hline \multirow{3}{*}{$\begin{array}{l}\text { IV } \\
20\end{array}$} & \multirow{3}{*}{$\begin{array}{c}V \\
20\end{array}$} & \multirow{3}{*}{$\begin{array}{l}\text { VI } \\
20\end{array}$} & \multicolumn{6}{|c|}{ R. Q. } \\
\hline & & & I & II & III & IV & V & VI \\
\hline & & & 20 & 20 & 20 & 20 & 20 & 20 \\
\hline 9.3 & 9.5 & 10.3 & 0.76 & 0.85 & 0.76 & 0.80 & 0.79 & 0.81 \\
\hline 13.6 & 8.8 & 13.8 & 0.92 & 0.85 & 0.81 & 0.83 & 0.77 & 0.85 \\
\hline 12.3 & 13.6 & 13.7 & 0.95 & 0.90 & 0.87 & 0.86 & 0.88 & 0.88 \\
\hline 13.0 & 12.9 & 10.7 & 0.84 & 0.64 & 0.81 & 0.82 & 0.84 & 0.82 \\
\hline 16.8 & 14.5 & 13.4 & 0.87 & 0.88 & 0.92 & 0.94 & 0.86 & 0.91 \\
\hline 11.1 & 8.5 & 12.0 & 0.81 & 0.82 & 0.82 & 0.85 & 0.85 & 0.85 \\
\hline 7.3 & 11.1 & 6.7 & 0.98 & 0.89 & 0.76 & 0.73 & 0.88 & 0.74 \\
\hline 11.3 & 9.0 & 12.9 & 0.87 & 0.81 & 0.66 & 0.81 & 0.75 & 0.85 \\
\hline 11.8 & 11.0 & 11.7 & 0.88 & $\mathbf{0 . 8 3}$ & 0.80 & $\mathbf{0 . 8 3}$ & 0.83 & 0.84 \\
\hline \pm 0.67 & \pm 0.65 & \pm 0.59 & \pm 0.02 & \pm 0.02 & \pm 0.02 & \pm 0.01 & \pm 0.01 & \pm 0.01 \\
\hline
\end{tabular}

2.

solution upon tissue respiration of kidney cortex in vitro.

solution with $20 \mathrm{mgm}$ per cent sodium lactate.

\begin{tabular}{|c|c|c|c|c|c|c|c|c|}
\hline \multirow{3}{*}{$\begin{array}{l}\text { IV } \\
20\end{array}$} & \multirow{3}{*}{$\begin{array}{c}V \\
20\end{array}$} & \multirow{3}{*}{$\begin{array}{l}\text { VI } \\
20\end{array}$} & \multicolumn{6}{|c|}{ R. Q. } \\
\hline & & & I & II & III & IV & V & VI \\
\hline & & & 20 & 20 & 20 & 20 & 20 & 20 \\
\hline 19.7 & 19.9 & 16.3 & 0.96 & 1.01 & 0.93 & 0.89 & 0.95 & 0.86 \\
\hline 12.4 & 11.5 & 12.6 & 0.80 & 0.79 & 0.80 & 0.77 & 0.78 & 0.82 \\
\hline 13.2 & 19.7 & 16.9 & 0.73 & 0.82 & 0.91 & 0.80 & 0.94 & 0.88 \\
\hline 11.6 & 9.5 & 11.5 & 0.81 & 0.79 & 0.82 & 0.82 & 0.77 & 0.80 \\
\hline 13.5 & 9.4 & 14.3 & 0.82 & 0.77 & 0.78 & 0.79 & 0.74 & 6.85 \\
\hline 20.0 & 30.4 & 27.7 & 0.84 & 0.89 & 0.64 & 0.84 & 0.92 & 0.97 \\
\hline 13.8 & 15.2 & 11.4 & 0.75 & 0.65 & 0.74 & 0.77 & 0.81 & 0.80 \\
\hline 13.5 & 11.3 & 11.0 & 0.01 & 0.98 & 0.93 & 0.90 & 0.84 & 0.86 \\
\hline 14.7 & 14.6 & 15.2 & 0.84 & 0.84 & 0.82 & 0.82 & 0.84 & 0.86 \\
\hline \pm 0.82 & \pm 1.74 & \pm 1.22 & \pm 0.02 & \pm 0.03 & \pm 0.03 & \pm 0.01 & \pm 0.02 & \pm 0.01 \\
\hline
\end{tabular}


(b) Tissue respiration of rabbit kidney cortex in

\begin{tabular}{|c|c|c|c|c|c|c|c|c|c|c|}
\hline \multirow{2}{*}{ No. } & \multirow{2}{*}{ Date } & \multicolumn{6}{|c|}{ Qo } & \multicolumn{3}{|c|}{$\mathbf{Q}$} \\
\hline & & $\underset{20}{I}$ & $\begin{array}{l}\text { II } \\
20\end{array}$ & $\begin{array}{c}\text { III } \\
20\end{array}$ & $\begin{array}{l}\text { IV } \\
20\end{array}$ & $\begin{array}{c}V \\
20\end{array}$ & $\begin{array}{l}\text { VI } \\
20\end{array}$ & $\begin{array}{c}\mathbf{I} \\
\mathbf{2 0}\end{array}$ & $\begin{array}{l}\text { II } \\
\mathbf{2 0}\end{array}$ & $\begin{array}{l}\text { III } \\
\mathbf{2 0}\end{array}$ \\
\hline 1 & $12 / 6$ & 24.4 & 20.5 & 18.8 & 18.8 & 16.6 & 10.1 & 22.3 & 18.8 & 16.4 \\
\hline 2 & $13 / 6$ & 30.6 & 16.8 & 14.3 & 18.1 & 18.2 & 13.2 & 27.0 & 13.5 & 11.3 \\
\hline 3 & $13 / 6$ & 22.9 & 19.0 & 15.3 & 15.0 & 13.9 & 15.2 & 21.0 & 17.5 & 13.2 \\
\hline 4 & $17 / 6$ & 18.7 & 13.1 & 13.7 & 9.9 & 9.8 & 11.6 & 18.0 & 10.8 & 11.6 \\
\hline 5 & $18 / 6$ & 14.1 & 23.8 & 17.0 & 17.4 & 18.3 & 16.5 & 12.2 & 22.1 & 14.9 \\
\hline 6 & $19 / 6$ & 25.2 & 18.8 & 16.3 & 16.4 & 15.4 & 14.7 & 22.5 & 17.4 & 14.1 \\
\hline 7 & $20 / 6$ & 38.0 & 30.6 & 26.7 & 24.5 & 18.3 & 18.1 & 36.5 & 28.9 & 24.8 \\
\hline 8 & $20 / 6$ & 21.3 & 16.1 & 8.1 & 9.1 & 7.3 & 13.8 & 21.7 & 15.9 & 7.6 \\
\hline & Mean & $\begin{array}{r}24.4 \\
+165\end{array}$ & $\begin{array}{r}19.8 \\
\end{array}$ & 16.3 & 16.2 & 14.7 & 14.2 & 22.7 & 18.1 & 14.2 \\
\hline & & \pm 1.65 & | 1.23 & \pm 1.09 & \pm 1.15 & \pm 1.05 & \pm 0.63 & $\| \pm 1.46$ & \pm 1.23 & \pm 1.07 \\
\hline
\end{tabular}

TABLE

Effect of 60 mgm per cent sodium lactate in Ring er's

(a) Tissue respiration of rabbit kidney cortex in Ring e r's

\begin{tabular}{|c|c|c|c|c|c|c|c|c|c|c|}
\hline \multirow{2}{*}{ No. } & \multirow{2}{*}{ Date } & \multicolumn{6}{|c|}{ Qo } & \multicolumn{3}{|c|}{ Qc } \\
\hline & & $\underset{20}{I}$ & $\begin{array}{l}\text { II } \\
20\end{array}$ & $\begin{array}{c}\text { III } \\
20\end{array}$ & $\begin{array}{l}\text { IV } \\
20\end{array}$ & $\begin{array}{c}\mathrm{V} \\
20\end{array}$ & $\begin{array}{l}\text { VI } \\
20\end{array}$ & $\begin{array}{c}\text { I } \\
20\end{array}$ & $\begin{array}{l}\text { II } \\
20\end{array}$ & $\begin{array}{c}\text { III } \\
20\end{array}$ \\
\hline 1 & $4 / 10$ & 25.5 & 26.0 & 21.9 & 16.5 & 14.9 & 14.0 & 22.2 & 22.4 & 18.2 \\
\hline 2 & $7 / 10$ & 33.1 & 27.4 & 22.2 & 16.7 & 13.1 & 14.5 & 31.8 & 23.5 & 19.3 \\
\hline 3 & $8 / 10$ & 19.0 & 17.2 & 17.6 & 15.0 & 18.4 & 15.6 & 15.4 & 12.6 & 13.6 \\
\hline 4 & $9 / 10$ & 27.6 & 15.9 & 22.6 & 18.1 & 13.2 & 15.5 & 27.0 & 13.1 & 17.8 \\
\hline 5 & $10 / 10$ & 20.0 & 18.4 & 20.1 & 13.2 & 11.0 & 16.4 & 15.3 & 13.4 & 15.7 \\
\hline 6 & $10 / 10$ & 16.8 & 14.3 & 20.4 & 18.6 & 13.5 & 18.9 & 12.0 & 9.3 & 14.3 \\
\hline 7 & $21 / 10$ & 19.0 & 20.9 & 20.6 & 16.7 & 15.5 & 17.6 & 14.1 & 16.3 & 15.7 \\
\hline & Mean & 23.0 & 20.0 & 20.8 & 16.4 & 14.2 & 16.1 & 19.7 & 15.8 & 16.4 \\
\hline & & \pm 1.70 & \pm 1.41 & \pm 0.43 & \pm 0.45 & \pm 0.60 & \pm 0.46 & \pm 2.16 & \pm 1.46 & \pm 0.61 \\
\hline
\end{tabular}

(b) Tissue respiration of rabbit kidney cortex in

\begin{tabular}{|c|c|c|c|c|c|c|c|c|c|c|}
\hline \multirow{2}{*}{ No. } & \multirow[b]{2}{*}{ Date } & \multicolumn{6}{|c|}{ Qo } & \multicolumn{3}{|r|}{$Q c$} \\
\hline & & $\begin{array}{c}\text { I } \\
20\end{array}$ & $\begin{array}{l}\text { II } \\
20\end{array}$ & $\begin{array}{c}\text { III } \\
20\end{array}$ & $\begin{array}{l}\text { IV } \\
20\end{array}$ & $\begin{array}{c}V \\
20\end{array}$ & $\begin{array}{l}\text { VI } \\
20\end{array}$ & $\begin{array}{c}I \\
20\end{array}$ & $\begin{array}{l}\text { II } \\
20\end{array}$ & $\begin{array}{c}\text { III } \\
\mathbf{2 0}\end{array}$ \\
\hline 1 & $4 / 10$ & 18.8 & 20.9 & 20.6 & 16.8 & 17.3 & 14.6 & 15.5 & 17.5 & 17.4 \\
\hline 2 & $7 / 10$ & 12.9 & 14.9 & 13.7 & 18.6 & 16.3 & 14.7 & 11.2 & 12.7 & 11.4 \\
\hline 3 & $8 / 10$ & 19.1 & 25.5 & 19.9 & 14.3 & 15.8 & 15.2 & 16.7 & 23.5 & 17.2 \\
\hline 4 & $9 / 10$ & 14.6 & 19.4 & 13.9 & 21.7 & 12.5 & 16.5 & 13.0 & 18.4 & 10.1 \\
\hline 5 & $10 / 10$ & 24.2 & 18.5 & 20.2 & 20.3 & 15.2 & 18.0 & 22.4 & 16.1 & 17.6 \\
\hline 6 & $10 / 10$ & 21.0 & 17.6 & 18.0 & 19.0 & 16.7 & 14.2 & 18.2 & 15.7 & 15.2 \\
\hline 7 & $21 / 10$ & 21.2 & 16.1 & 15.8 & 13.9 & 13.0 & 10.3 & 18.6 & 13.0 & 12.9 \\
\hline & Mean & 18.8 & 19.0 & 17.4 & 17.8 & 15.3 & 14.8 & 16.5 & 16.7 & 14.5 \\
\hline & & \pm 1.01 & \pm 0.87 & \pm 0.88 & \pm 0.83 & \pm 0.50 & \pm 0.53 & \pm 0.97 & \pm 0.92 & \pm 0.91 \\
\hline
\end{tabular}


the normal Ringer's solution.

\begin{tabular}{|c|c|c|c|c|c|c|c|c|}
\hline \multirow[b]{2}{*}{$\begin{array}{l}\text { IV } \\
20\end{array}$} & \multirow[b]{2}{*}{$\begin{array}{l}V \\
20\end{array}$} & \multirow[b]{2}{*}{$\begin{array}{l}\text { VI } \\
20\end{array}$} & \multicolumn{6}{|c|}{ R. Q. } \\
\hline & & & $\begin{array}{c}I \\
20\end{array}$ & $\begin{array}{l}\text { II } \\
20\end{array}$ & $\begin{array}{c}\text { III } \\
20\end{array}$ & $\begin{array}{l}\text { IV } \\
20\end{array}$ & $\begin{array}{c}V \\
20\end{array}$ & $\begin{array}{l}\text { VI } \\
20\end{array}$ \\
\hline 16.6 & 14.1 & 8.2 & 0.91 & 0.92 & 0.87 & 0.88 & 0.85 & 0.81 \\
\hline 14.8 & 15.2 & 7.6 & 0.88 & 0.80 & 0.79 & 0.82 & 0.84 & 0.58 \\
\hline 13.0 & 12.2 & 13.3 & 0.92 & 0.92 & 0.86 & 0.87 & 0.88 & 0.88 \\
\hline 8.1 & 7.7 & 9.6 & 0.96 & 0.82 & 0.85 & 0.82 & 0.79 & 0.83 \\
\hline 15.5 & 16.7 & 15.1 & 0.87 & 0.93 & 0.88 & 0.89 & 0.91 & 0.92 \\
\hline 13.9 & 13.4 & 13.6 & 0.89 & 0.93 & 0.87 & 0.85 & 0.87 & 0.93 \\
\hline 22.2 & 15.9 & 16.6 & 0.96 & 0.95 & 0.93 & 0.90 & 0.87 & 0.92 \\
\hline 7.4 & 5.9 & 7.2 & 0.82 & 0.99 & 0.94 & 0.81 & 0.81 & 0.52 \\
\hline 13.9 & 12.6 & 11.4 & 0.90 & 0.91 & 0.87 & 0.86 & 0.85 & 0.80 \\
\hline \pm 1.07 & \pm 0.97 & \pm 1.04 & \pm 0.01 & \pm 0.02 & \pm 0.01 & \pm 0.01 & \pm 0.01 & \pm 0.04 \\
\hline
\end{tabular}

3.

solution upon tissue respiration of kidney cortex in vitro.

solution with $60 \mathrm{mgm}$ per cent sodium lactate.

\begin{tabular}{|c|c|c|c|c|c|c|c|c|}
\hline & & & \multicolumn{6}{|c|}{ R. Q. } \\
\hline IV & $\mathrm{V}$ & VI & I & II & III & IV & V & VI \\
\hline 20 & 20 & 20 & 20 & 20 & 20 & 20 & 20 & 20 \\
\hline 12.1 & 10.8 & 10.4 & 0.87 & 0.86 & 0.83 & 0.73 & 0.73 & 0.74 \\
\hline 13.5 & 10.0 & 11.9 & 0.93 & 0.86 & 0.87 & 0.81 & 0.77 & 0.82 \\
\hline 11.8 & 15.3 & 13.1 & 0.81 & 0.73 & 0.77 & 0.79 & 0.83 & 0.84 \\
\hline 14.7 & 9.7 & 12.4 & 0.98 & 0.83 & 0.79 & 0.81 & 0.74 & 0.80 \\
\hline 9.2 & 7.5 & 13.2 & 0.77 & 0.73 & 0.78 & 0.70 & 0.68 & 0.80 \\
\hline 14.5 & 10.3 & 15.8 & 0.72 & 0.65 & 0.70 & 0.78 & 0.76 & 0.84 \\
\hline 12.6 & 11.7 & 14.6 & 0.74 & 0.78 & 0.76 & 0.75 & 0.75 & 0.83 \\
\hline 12.6 & 10.8 & 13.1 & 0.83 & 0.78 & $\begin{array}{l}0.79 \\
\end{array}$ & 0.77 & 0.75 & 0.81 \\
\hline \pm 0.47 & \pm 0.55 & \pm 0.44 & \pm 0.03 & \pm 0.02 & \pm 0.01 & \pm 0.01 & \pm 0.01 & \pm 0.01 \\
\hline
\end{tabular}

the normal Ring e r's solution.

\begin{tabular}{|c|c|c|c|c|c|c|c|c|}
\hline & & & \multicolumn{6}{|c|}{ R. Q. } \\
\hline IV & V & VI & I & II & III & IV & V & VI \\
\hline 20 & 20 & 20 & 20 & 20 & 20 & 20 & 20 & 20 \\
\hline 13.5 & 14.8 & 11.9 & 0.82 & 0.84 & 0.84 & 0.80 & 0.86 & 0.82 \\
\hline 16.3 & 15.6 & 13.4 & 0.87 & 0.85 & 0.83 & 0.88 & 0.96 & 0.91 \\
\hline 12.0 & 13.3 & 13.2 & 0.87 & 0.92 & 0.86 & 0.84 & 0.84 & 0.87 \\
\hline 18.8 & 9.6 & 13.0 & 0.89 & 0.95 & 0.73 & 0.87 & 0.77 & 0.79 \\
\hline 18.2 & 13.0 & 15.5 & 0.93 & 0.87 & 0.87 & 0.90 & 0.86 & 0.86 \\
\hline 16.0 & 14.6 & 11.6 & 0.87 & 0.89 & 0.84 & 0.84 & 0.87 & 0.82 \\
\hline 11.2 & 10.7 & 8.2 & 0.88 & 0.81 & 0.82 & 0.81 & 0.82 & 0.80 \\
\hline 15.1 & 13.1 & 12.4 & 0.88 & 0.88 & 0.83 & 0.85 & 0.85 & 0.84 \\
\hline \pm 0.86 & \pm 0.59 & \pm 0.54 & \pm 0.01 & \pm 0.01 & \pm 0.01 & \pm 0.01 & \pm 0.01 & \pm 0.01 \\
\hline
\end{tabular}




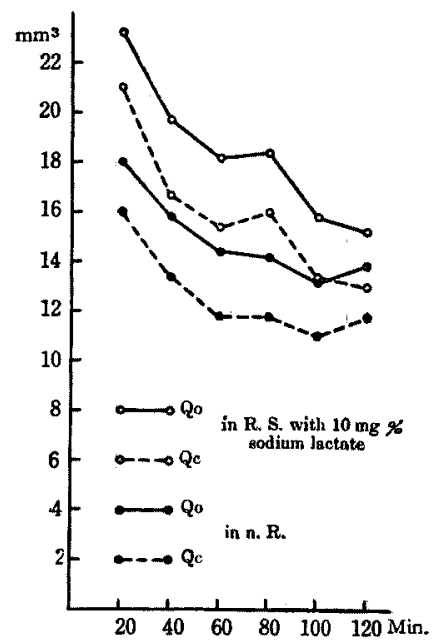

Fig. 1. Comparison of the tissue respiration of rabbit kidney cortex in Ringer's solution with $10 \mathrm{mgm}$ per cent sodium lactate and in the normal $R$ inger's solution.

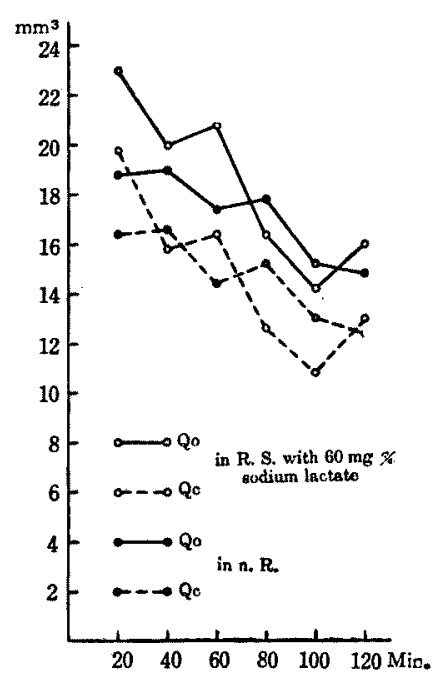

Fig. 3. Comparison of the tissue respiration of rabbit kidney cortex in $R$ inger's solution with $60 \mathrm{mgm}$ per cent sodium lactate and in the normal Ringer's solution.

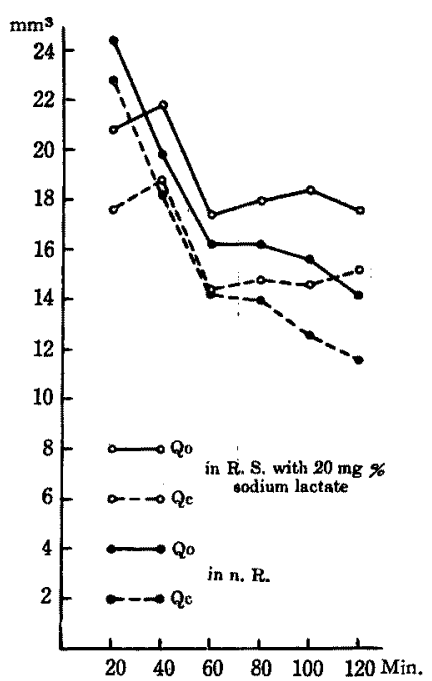

Fig. 2. Comparison of the tissue respiration of rabbit kidney cortex in Ringer's solution with $20 \mathrm{mgm}$ per cent sodium lactate and in the normal Ringer's solution.

The means of the oxygen consumption Qo and carbon dioxide output Qc of Table 3 a for two hours were 18.4 and 14.7 $\mathrm{cmm}$ respectively, of which the means for the first hour were 21.3 and $17.3 \mathrm{cmm}$ and those for the second 15.6 and $12.2 \mathrm{cmm}$.

The means of Qo and Qc of Table $3 \mathrm{~b}$ (control experiment for Table 3 a) for two hours were 17.2 and $14.7 \mathrm{cmm}$ respectively, of which the means for the first hour were 18.4 and $15.9 \mathrm{cmm}$ and those for the second 15.6 and $13.5 \mathrm{cmm}$.

As the above figures show, the difference caused by addition of $60 \mathrm{mgm}$ per cent sodium lactate to Ring er's solution was quite small and within the limit of experimental errors.

R. Q. were relatively higher in the normal Ringer's solution than in the lactate-Ringer's solution. 


\section{Concluding Remarks.}

Since Meyerh of and others ${ }^{455}$ published their reports on the relation between lactate and tissue respiration, several investigators have studied on this subject.

Gerard $\mathrm{d}^{\mathrm{s})}$ observed that sodium lactate, 0.1 per cent, had no effect on tissue respiration of nerve, though stronger solution caused some decrease. The sodium lactate concentration of 0.1 per cent is higher than the highest concentration of $60 \mathrm{mgm}$ per cent in our case, with which we found only a slight change of the tissue respiration of kidney cortex. Loebel and Hickling, however,") in their studies on excised testicular tissue of rat observed that with $\mathrm{m} / 40$ (about 0.28 per cent) sodium lactate in the medium the oxygen consumption was greater and remained at almost the same level for several hours and that the R. Q. showed a definite rise. Richard son and his coworkers ${ }^{10}$ found that the sections of dog kidney were influenced by sodium lactate $(0.01 \mathrm{~mol})$ resulting in raising the oxygen consumption and respiratory quotient. The increase of respiration amounted to $44 \%$. D i ckens and $\breve{S}$ im er ${ }^{11)}$ found in their experiments on liver tissue a definitely high $R$. Q. in addition to an increased respiration in the presence of lactate $(\mathrm{m} / 45)$. The $y^{12)}$ confirmed again later that the concentration of lactate in the serum was sufficient to cause a marked consumption of this substance by kidney tissue in serum. $\mathrm{Ki} \mathrm{s} \mathrm{h}^{13)}$ also found an increased oxygen consumption of kidney and liver tissues by addition of $\mathrm{m} / 50-\mathrm{m} / 5000$ sodium lactate. The increase amounted to $10-50 \%$ in this case. He extended his studies on various other tissues and obtained more remarkable influences of a similar nature. Sh affer and others ${ }^{14)}$ observed that sodium lactate (100 mgm per cent) added to $\mathrm{R}$ in g e r's solution produced no appreciable effect on nerve tissue respiration.

Some of the above cited investigators have shown that the tissue respiration was increased with relatively higher concentration $(\mathrm{m} / 40)$ of sodium lactate in Ringer's solution. "Our own results summarized in table 1, 2 and 3 show, however, that the increased extent of respiration was the most remarkable when sodium lactate concentration was $10 \mathrm{mgm}$ per cent. With concentration of $20 \mathrm{mgm}$ per cent the increase was less, and with that of $60 \mathrm{mgm}$ per cent there was some appreciable

8) Gerard, R. W.; Am. J. Physiol., 1927, 82, 381.

9) Loebel, R. O. and R. A. Hickling; ibid., 1927, 81, 494.

10) Richardson, H. B., E. Shorr and R. O. Loebel; J. Biol. Chem., 1930, 86, 551.

11) Dickens, F. and F. Š imer; Biochem. J,, 1930, 24, 1301.

12) Id. ; ibid., 1931, 25, 11.

13) K i s c h, B.; Biochem. Z., 1932, 253, 346.

14) Shaffer, M., T. H. Chang and R. W. Gerard ; Am. J. Physiol., 1935, 111, 697. 
accerelating effect on the tissue respiration at the beginning of the experimental period. Against the remarks of other investigators respiratory quotient was not raised by addition of sodium lactate in our case.

At any rate, as the addition of $10 \mathrm{mgm}$ per cent sodium lactate to $R$ inger's solution caused a remarkable and $20 \mathrm{mgm}$ per cent a somewhat less remarkable increase of the rate of the tissue respiration in vitro (these concentrations being considered to be physiological ${ }^{15}$ ), we are sure that the lactate is one of the factors which cause higher rate of tissue respiration in serum than in Ring er's solution.

\section{Summary.}

By Warburg's second method ${ }^{2)}$ with our modification effect of sodium lactate on tissue respiration of rabbit kidney cortex was investigated.

Sodium lactate was added to Ringer's solution in concentrations' of 10,20 and $60 \mathrm{mgm}$ per cent.

With $10 \mathrm{mgm}$ per cent the greatest increase was observed which amounted to $20-30 \%$ of the normal value.

With $20 \mathrm{mgm}$ per cent the increase was a little less and with 60 mgm per cent we could find some accerelating effect at the beginning of the experimental period.

16) Oinuma, S. ; Japanese Anatomical and Physiological Constants and Data (Jap.), 1934, Okayama, 136. 